\title{
Highjacking of PI3K/AKT signaling pathway by Hepatitis $C$ virus in TLR9-activated human plasmacytoid dendritic cells
}

\author{
Jonathan Florentin', Clélia Dental', Guylène Firaguay', Françoise Gondois-Rey', Vassili Soumelis², \\ Thomas F Baumert ${ }^{3}$, Jacques A Nunès ${ }^{1}$, Daniel Olive ${ }^{1}$, Ivan Hirsch ${ }^{1 *}$
}

From $16^{\text {th }}$ International Symposium on HIV and Emerging Infectious Diseases Marseille, France. 24-26 March 2010

\section{Background}

Plasmacytoid dendritic cells (pDCs) are responsible for the production of type I IFN during viral infection. Viral elimination by IFN-alpha-based therapy in more than $50 \%$ of patients chronically infected with hepatitis $C$ virus (HCV) suggests a possible impairment of production of endogenous IFN-alpha by pDCs in infected individuals. Recent studies in the HCVcc-exposed pDCs purified from healthy donors show that $\mathrm{HCV}$ is a weak inducer of IFN-alpha in vitro and that HCVcc blocks the TLR9mediated IFN-alpha production. It has been also reported that PI3K/AKT is critical for type I IFN production by pDCs in response to TLR agonists. The specific aim of the present study is to investigate the effect of $\mathrm{HCV}$ on PI3K/AKT signaling.

\section{Methods}

To this end we exposed pDCs from healthy donors to insect cell-derived HCV-like particles (HCV-LP) or an insect cell control preparation in the presence or absence of TLR7 and TLR9 agonists and determined dynamics of PI3K/AKT phosphorylation by flow cytometry. By this approach we compared the early (AKT phosphorylation) and late (IFN-alpha production) steps of TLR7/TLR9-MyD88 signaling. The levels of cell-free supernatant-secreted IFN-alpha were determined by ELISA.

\section{Results}

Expression of TLR9 gene was analysed by quantitative RT-PCR. Whereas phosphorylation of AKT increased 4 times during 12-h culture of pDCs in the presence of IL-3 and it was increased further by $50 \%$ after stimulation with CpG-C, it dropped-down to the basal level, when pDCs were preincubated with HCV-LP. Expression of TLR9 during 12-h culture of $\mathrm{pDCs}$ in the presence of IL-3 was reduced $10^{4}$ times, whereas it was reduced $10^{6}$ times, when pDCs were stimulated with CpG-C. HCV-LP did not show any silencing effect on TLR9 expression.

\section{Discussion}

We conclude that HCV-LP block the TLR9-mediated IFN-alpha production upstream of PI3K/AKT pathway and that HCV-LP do not block transcription of TLR9 gene. These findings suggest that HCV impairs signalization via TLR9 upstream of PI3K/AKT pathway in pDCs. Furthermore, our model system will allow elucidating the mechanism of the blockade of TLR9 signaling by $\mathrm{HCV}$ in pDCs. (ANRS grant 2007/306).

\section{Author details}

'INSERM, UMR891, Centre de Recherche en Cancérologie de Marseille and Institut Paoli-Calmettes, and Université Méditerranée, Marseille, France. ${ }^{2}$ Institut Curie, Paris, France. ${ }^{3}$ INSERM UMR_S748, Université de Strasbourg, Strasbourg, France.

Published: 11 May 2010

doi:10.1186/1742-4690-7-S1-P7

Cite this article as: Florentin et al.: Highjacking of PIBK/AKT signaling pathway by Hepatitis C virus in TLR9-activated human plasmacytoid dendritic cells. Retrovirology 2010 7(Suppl 1):P7.

* Correspondence: ivan.hirsch@inserm.fr

'INSERM, UMR891, Centre de Recherche en Cancérologie de Marseille and Institut Paoli-Calmettes, and Université Méditerranée, Marseille, France 